\title{
In vitro activity of fosfomycin in combination with colistin against clinical isolates of carbapenem- resistant Pseudomas aeruginosa
}

\author{
Xiuzhen $\mathrm{Di}^{1}$, Rui Wang ${ }^{1}$, Bin $\mathrm{Liu}^{2}$, Xin Zhang ${ }^{2}$, Wentao $\mathrm{Ni}^{2}$, Jin Wang ${ }^{1}$, Beibei Liang ${ }^{1}$, Yun $\mathrm{Cai}^{1}$ and \\ Youning Liu $^{2}$
}

The shortage of effective antibiotics against carbapenem-resistant Pseudomonas aeruginosa (CRPA) poses a public health threat. Combination treatment may represent a good choice for treating infections caused by CRPA. The aim of this study was to evaluate the in vitro efficacy of fosfomycin in combination with colistin against clinical CRPA isolates. Eighty-seven isolates were collected from three hospitals in China. The checkerboard method and time-kill assay were used to assess the interactions between fosfomycin and colistin. The fosfomycin/colistin combination displayed synergistic and partial synergistic activity against $21.84 \%$ and $27.59 \%$ of the isolates, respectively. Antagonism was not observed. In combination, the colistin MIC values were $\leqslant 0.5 \mu \mathrm{g} \mathrm{ml}^{-1}$ for $91.95 \%$ of the isolates. This result differed significantly from those obtained using a single agent treatment (The colistin MIC values were $\leqslant 0.5 \mu \mathrm{g} \mathrm{ml}^{-1}$ for only $25.29 \%$ of the isolates). In addition, the time-kill assay demonstrated that the fosfomycin/colistin combination treatment exerted bactericidal effects against five isolates and that the regrowth observed after colistin monotherapy was prevented. In summary, the combination of fosfomycin and colistin demonstrated synergistic activity against the CRPA isolates tested in this study. Furthermore, fosfomycin may potentially widen the therapeutic window of colistin, suggesting that this combination could be applied clinically to control infections caused by CRPA.

The Journal of Antibiotics (2015) 68, 551-555; doi:10.1038/ja.2015.27; published online 25 March 2015

\section{INTRODUCTION}

Pseudomonas aeruginosa (PA) is an important pathogen that causes serious healthcare-associated infections. Carbapenem is the mainstay for the empirical treatment of suspected $P$. aeruginosa infections due to its excellent antimicrobial activity. ${ }^{1}$ However, outbreaks and the increased prevalence of carbapenem-resistant PA (CRPA) have been continuously reported in hospitals worldwide, resulting in high morbidity, mortality, and medical costs. ${ }^{2}$ CRPA pathogens can confer resistance not only to most clinically available $\beta$-lactam agents but also to other classes of antibiotics such as aminoglycosides, fluoroquinolones, and co-trimoxazole. ${ }^{3}$ Consequently, this problem renders the empirical choice of an appropriate antimicrobial treatment very difficult. $^{4}$

Colistin, or polymyxin $\mathrm{E}$, is a cationic polypeptide antibiotic that was discovered $>60$ years ago. Because of its nephrotoxicity and neurotoxicity, the use of colistin has been discontinued since the 1970s. ${ }^{5}$ Currently, colistin is typically used to treat infections of multidrug-resistant $P$. aeruginosa (MDR-PA). ${ }^{6}$ However, antibiotic resistance and heteroresistance have also been associated with colistin treatment, particularly if the drug is used as a monotherapy or at a high dosage. ${ }^{7}$ This finding highlights the importance of investigating rational combinations of colistin and other antibiotics for the treatment of CRPA infections. ${ }^{8}$ Fosfomycin, a phosphonic acid derivative discovered in Spain in 1969, exhibits bactericidal activity against a wide range of bacteria, including Gram-negative bacteria (GNB) and some Gram-positive bacteria. In recent years, the drug has evoked interest as a treatment for both urinary and systemic infections arising from GNB that are resistant to traditional agents because it appears to retain antimicrobial activity against a substantial percentage of these isolates. ${ }^{9}$

In this study, we investigated the in vitro activity of the combination treatment of colistin and fosfomycin against CRPA isolates. We further determined whether such a combination could potentially be used in the clinic for treating CRPA infections.

\section{MATERIALS AND METHODS}

\section{Bacterial strains}

Eighty-seven non-duplicate clinical isolates of CRPA were collected between February 2012 and March 2013 from three hospitals in Beijing, China (PLA General Hospital 22 strains, Beijing Hospital 27 strains, Navy General Hospital 38 strains) in this study. Sixty-five strains were collected from sputum, 13 from urine, 4 from wound sites, 3 from catheters, and 2 from blood. The

\footnotetext{
${ }^{1}$ The Center of Medicine Clinical Research, Translational Medical Center of Chinese People's Liberation Army General Hospital, Beijing, China and ${ }^{2}$ Department of Respiratory Diseases, Chinese People's Liberation Army General Hospital, Beijing, China

Correspondence: Professor R Wang, The Center of Medicine Clinical Research, Translational Medical Center of Chinese People's Liberation Army General Hospital, NO. 28, Fuxing Ro, Beijing 100853, China.

E-mail: wangruivip301@sina.com

Received 31 May 2014; revised 9 February 2015; accepted 21 February 2015; published online 25 March 2015
} 
identification and antimicrobial susceptibility profiles of the isolates were determined with the VITEK-2 compact system using the cards ID-GNB and AST-GN09 (BioMérieux, Marcy l'Etoile, France). Quality control was assessed using the strain $P$. aeruginosa ATCC 27853 (ATCC, Manassas, VA, USA).

\section{Antibiotics and other materials}

Fosfomycin powder (96.5\% purity) was obtained from the National Institute for the Control of Pharmaceutical and Biological Products (NICPBP, Beijing, China). Colistin sulfate powder with a potency of $23584 \mathrm{Umg}^{-1}$ and D-glucose-6-phosphate disodium salt hydrate (G-6-P) were purchased from Sigma-Aldrich (St Louis, MO, USA). Mueller Hinton agar (MHA) and cationadjusted Mueller II broth (CA-MHB) were purchased from Becton, Dickinson (Franklin Lakes, NJ, USA). Fosfomycin and colistin were diluted in sterile water and used as prepared stock solutions of $2048 \mu \mathrm{g} \mathrm{ml}^{-1}$ and $1024 \mu \mathrm{g} \mathrm{ml}^{-1}$, respectively.

\section{MIC determination and synergistic activity testing}

The disk agar diffusion method was further used to verify antibiotic susceptibility with Oxoid discs (Oxoid, Wade Road, UK) containing imipenem $(10 \mu \mathrm{g})$, meropenem $(10 \mu \mathrm{g})$, amikacin $(30 \mu \mathrm{g})$, gentamicin $(10 \mu \mathrm{g})$, ceftazidime $(30 \mu \mathrm{g})$, cefepime $(30 \mu \mathrm{g})$, aztreonam $(30 \mu \mathrm{g})$, ciprofloxacin $(5 \mu \mathrm{g})$ or piperacillintazobactam $(100 \mu \mathrm{g} / 10 \mu \mathrm{g})$ placed on MHA, according to the Clinical and Laboratory Standards Institute's (CLSI) recommendations. ${ }^{10}$ The MIC values for fosfomycin were determined by the agar dilution method supplemented with $25 \mu \mathrm{g} \mathrm{ml}^{-1}$ G-6-P, and the MIC values for colistin were evaluated using the broth microdilution method, according to the CLSI's recommendations. ${ }^{10}$ P. aeruginosa ATCC 27853 was included in each method as the quality control strain. The MIC was defined as the lowest drug concentration that inhibited the visible growth of colonies. CRPA was defined as those isolates that were resistant to imipenem, according to the CLSI. ${ }^{10}$ MDR-PA isolates were defined as those that were non-susceptible to one or more agents in three or more antimicrobial categories. ${ }^{11}$ The experiments were performed in triplicate on separate days.

Synergy testing was performed in 96-well microplates with CA-MHB containing the tested agents at two-fold dilutions dispensed in a checkerboard pattern. The concentration ranges of these antibiotics were determined according to the MIC values obtained in the preliminary susceptibility tests described above. Fosfomycin supplemented with G-6-P $\left(25 \mu \mathrm{g} \mathrm{ml}^{-1}\right)$ was prepared in a checkerboard conformation. Fifty microliters of each drug and $100 \mu \mathrm{l}$ of the bacterial suspensions were added to the 96-well microplates. The final concentration of the inocula was approximately $1.5 \times 10^{5} \mathrm{CFU}$ per $\mathrm{ml}$ in each well. The MIC values of colistin determined using $P$. aeruginosa ATCC 27853 were similar in CA-MHB with and without G-6-P. The experiments were performed in triplicate on separate days. The interaction between the combinations of two study agents was determined according to the fractional inhibitory concentration index (FICI). The FICI for a given isolate was calculated as follows: $\mathrm{FICI}=$ (MIC of fosfomycin in combination/MIC of fosfomycin alone)+(MIC of colistin in combination/MIC of colistin alone). Synergy, partial synergy, additive, indifference, and antagonism were defined by FICI indices of $\leqslant 0.5,0.5<\mathrm{FICI}<1, \mathrm{FICI}=1,1<\mathrm{FICI} \leqslant 2$, and $\mathrm{FICI}>2$, respectively. ${ }^{12}$ The cumulative inhibition ratio (CIR) was defined as the percentage of isolates that were inhibited at a certain antibiotic concentration.

\section{Time-kill curves}

Twenty-four-hour time-kill assays were performed in duplicate to evaluate the combination of fosfomycin and colistin against five isolates (PA-1-5), for which synergy was demonstrated using the checkerboard method. Each isolate was treated with each antibiotic alone and in combination at its respective $1 / 2 \times$ MIC and $1 \times$ MIC values. The bacterial strains were inoculated at $\sim 5 \times 10^{5}$ CFU per ml into tubes containing freshly prepared CA-MHB supplemented with antibiotics, for a final volume of $10 \mathrm{ml}$ and were incubated at $37^{\circ} \mathrm{C}$. A tube without antibiotic was included in each experiment as a growth control. Aliquots were obtained from each tube at $0,2,4,8,12$, and $24 \mathrm{~h}$ after inoculation and were serially diluted and/or used as undiluted samples for determining viable counts. The diluted samples $(50 \mu \mathrm{l})$ were plated onto duplicate MHA plates, and then the plates were incubated at $37^{\circ} \mathrm{C}$ for $18 \mathrm{~h}$ in ambient air. The number of colonies that formed were counted, and the total bacterial $\log _{10} \mathrm{CFU}$ per $\mathrm{ml}$ of the original samples was calculated. Synergy was defined as $a \geqslant 100$-fold or $2-\log _{10}$ decrease, whereas antagonism was defined as $a \geqslant 100$-fold or $2-\log _{10}$ increase, in colony counts at $24 \mathrm{~h}$ induced by the treatment combination, compared with the most active single agent. ${ }^{13}$ Bactericidal activity of the single antibiotics or combinations was defined as a $\geqslant 1000$-fold or $3-\log _{10}$ reduction relative to the initial inocula after $24 \mathrm{~h}$ of incubation. ${ }^{14}$

\section{RESULTS}

\section{Susceptibility}

The antibiogram results demonstrated that $100 \%$ of the isolates were resistant to imipenem, $100 \%$ were resistant to ceftazidime and cefepime, $73.56 \%$ were resistant to meropenem, $51.73 \%$ were resistant to piperacillin-tazobactam, $45.98 \%$ were resistant to ciprofloxacin, $41.38 \%$ were resistant to gentamicin, $40.23 \%$ were resistant to aztreonam, and $39.08 \%$ were resistant to amikacin. Approximately $67 \%$ of the isolates were MDR-PA.

The MIC values for colistin and fosfomycin ranged from 0.125 $\mu \mathrm{g} \mathrm{ml}^{-1}$ to $4 \mu \mathrm{g} \mathrm{ml}^{-1}$ and $8 \mu \mathrm{g} \mathrm{ml}^{-1}$ to $512 \mu \mathrm{g} \mathrm{ml}^{-1}$, respectively. The colistin MIC values of $25.29 \%(22 / 87)$ of the isolates were $\leqslant 0.5 \mu \mathrm{g} \mathrm{ml}^{-1}$. The $\mathrm{MIC}_{50}$ and $\mathrm{MIC}_{90}$ values for fosfomycin were $64 \mu \mathrm{g} \mathrm{ml}^{-1}$ and $256 \mu \mathrm{g} \mathrm{ml}^{-1}$, respectively, whereas those of colistin

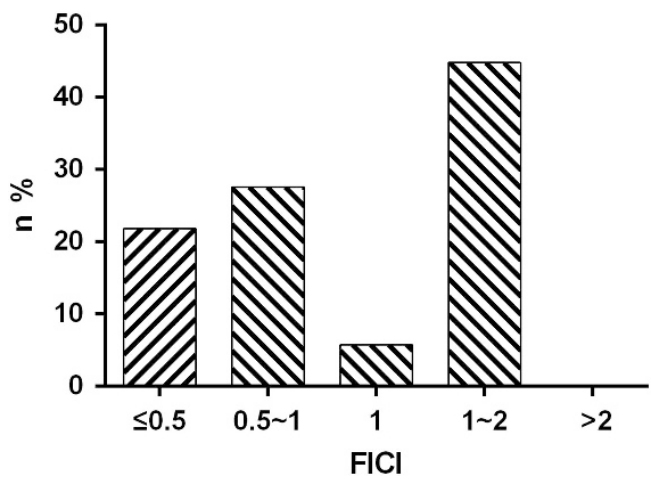

Figure 1 Fractional inhibitory concentration index $(\mathrm{FICl})$ for the combination treatment of fosfomycin and colistin against carbapenem-resistant Pseudomas aeruginosa $(n=87)$. Synergy, partial synergy, additive, indifference, and antagonism were defined by $\mathrm{FICl}$ indices of $\leqslant 0.5$, $0.5<\mathrm{FICl}<1, \mathrm{FICl}=1,1<\mathrm{FICl} \leqslant 2$, and $\mathrm{FICl}>2$, respectively.

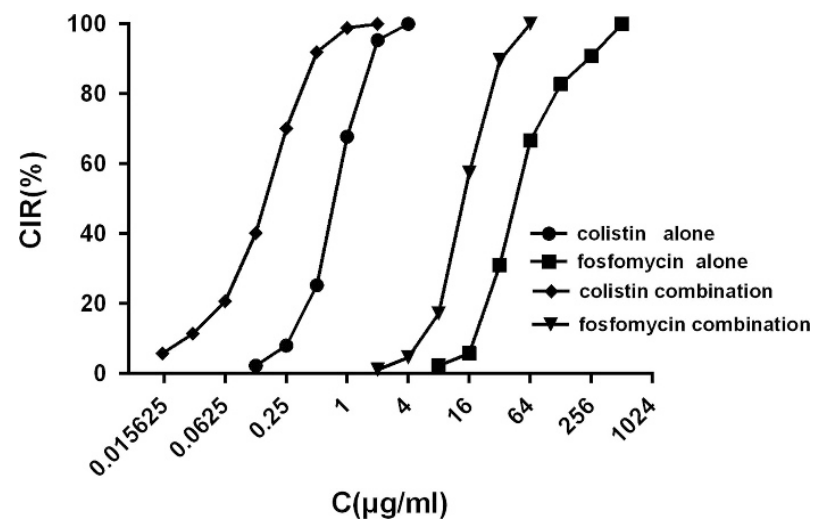

Figure 2 The cumulative inhibition ratio (CIR) of fosfomycin and/or colistin against carbapenem-resistant Pseudomas aeruginosa $(n=87)$. The CIR was defined as the percentage of isolates that were inhibited at a certain antibiotic concentration, C, concentration. 
were $1 \mu \mathrm{g} \mathrm{ml}^{-1}$ and $2 \mu \mathrm{g} \mathrm{ml}^{-1}$, respectively. Of the isolates analyzed, $95.40 \%(83 / 87)$ were susceptible to colistin.

Synergistic activity of the combination treatment

As shown in Figure 1, the drug combination displayed synergistic and partial synergistic activity in $21.84 \%$ (19/87) and $27.59 \%$ (24/87) of the isolates, respectively. For the other isolates, the combination treatment demonstrated additive or indifferent activity. Antagonism was not observed in this study. The MIC values of colistin and fosfomycin in combination ranged from $0.015 \mu \mathrm{g} \mathrm{ml}^{-1}$ to $2 \mu \mathrm{g} \mathrm{ml} l^{-1}$ and $2 \mu \mathrm{g} \mathrm{ml}^{-1}$ to $64 \mu \mathrm{g} \mathrm{ml}^{-1}$, respectively. The $\mathrm{MIC}_{50}$ and $\mathrm{MIC}_{90}$ were $0.25 \mu \mathrm{g} \mathrm{ml}^{-1}$ and $0.5 \mu \mathrm{g} \mathrm{ml}^{-1}$ for colistin, respectively, and 16 $\mu \mathrm{g} \mathrm{ml}^{-1}$ and $64 \mu \mathrm{g} \mathrm{ml}^{-1}$ for fosfomycin, respectively. The colistin MIC values were $\leqslant 0.5 \mu \mathrm{g} \mathrm{ml}^{-1}$ for $91.95 \%$ (80/87) of the isolates. The CIR curves for fosfomycin and/or colistin against CRPA are displayed in Figure 2. The CIR curves for both antibiotics were shifted to the left when the drugs were used in combination, compared with each drug alone, which suggests the potential utility of this combination.

Time-kill assay

The five CRPA isolates studied were MDR-PA with different MIC values for fosfomycin and colistin. As shown in Figure 3, the time-kill assays indicated that neither of the two agents demonstrated notable bactericidal activity against any of the five isolates when tested alone. Colistin monotherapy demonstrated bacteriostatic activity against PA-1, PA-2, PA-3, and PA- 4 at $1 \times$ MIC within $4 \mathrm{~h}$, but considerable regrowth occurred after $4 \mathrm{~h}$. However, when colistin was combined with fosfomycin, the regrowth did not occur, even after $24 \mathrm{~h}$ of incubation (Figure 3a-d). The combination of fosfomycin and colistin demonstrated bactericidal activity within $12 \mathrm{~h}$ at both $1 / 2 \times$ MIC and
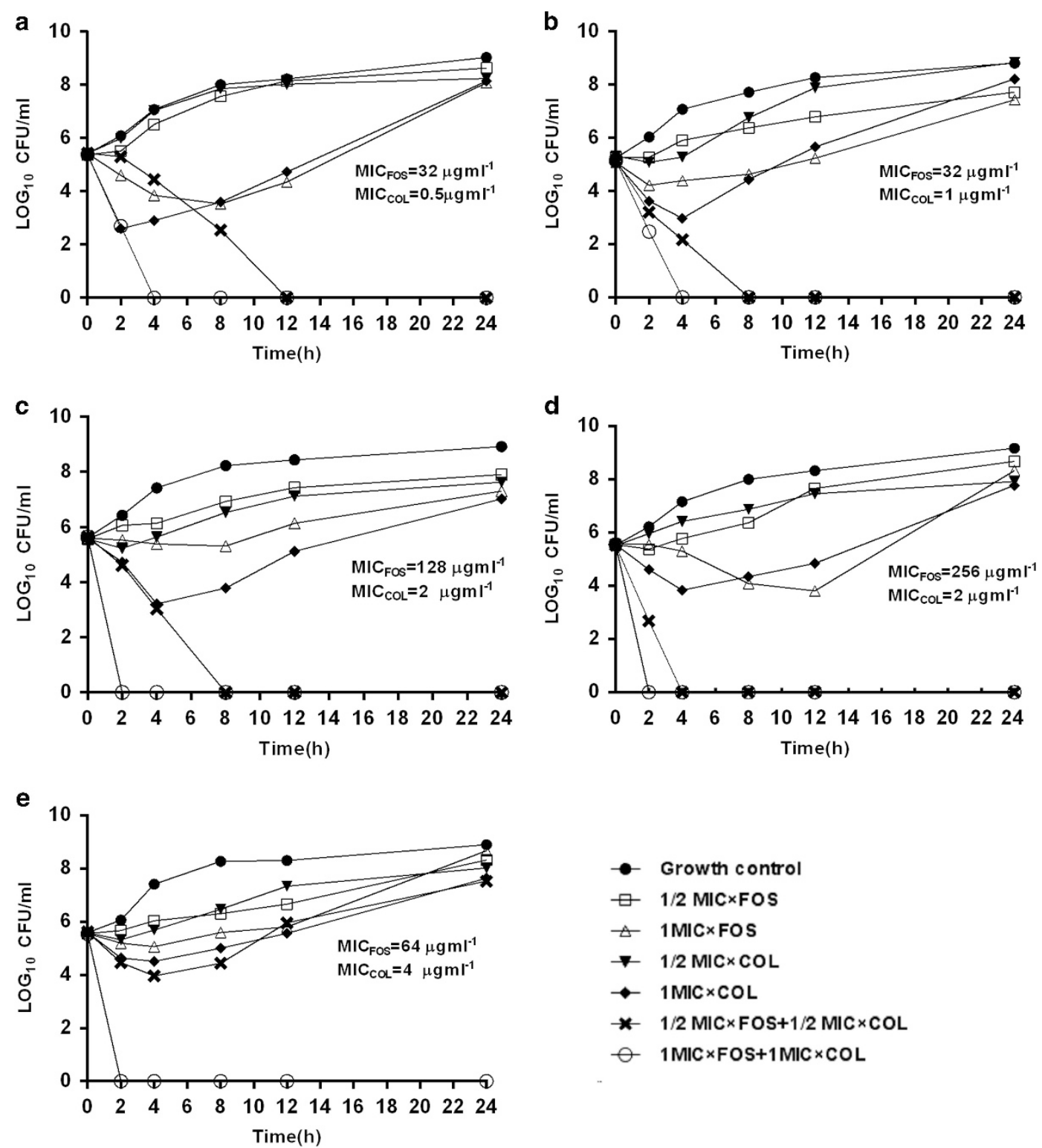

Figure 3 The time-kill curves of fosfomycin and/or colistin against carbapenem-resistant Pseudomas aeruginosa. The in vitro time-kill experiments were performed in duplicate, and the mean values were plotted. For the duplicate experiments, similar time-kill results were obtained. (a) $P$. aeruginosa-1 (PA-1); (b) P. aeruginosa- 2 (PA-2); (c) P. aeruginosa-3 (PA-3); (d) P. aeruginosa-4 (PA-4); (e) P. aeruginosa- 5 (PA-5). COL, colistin; FOS, fosfomycin. 
$1 \times$ MIC for PA-1, PA-2, PA-3, and PA-4 (Figure 3a-d), whereas the combination exhibited synergistic bactericidal activity for PA-5 only at $1 \times$ MIC (Figure $3 \mathrm{e}$ ).

\section{DISCUSSION}

The treatment of infections caused by $P$. aeruginosa is often difficult due to the natural and acquired resistance of this pathogen to several antibiotics. The emergence and global spread of CRPA is a major therapeutic and epidemiological challenge that leaves few available therapeutic options. In this study, all CRPA isolates were resistant to the antibiotics tested, including ceftazidime, aztreonam, and ciprofloxacin, etc., to various degrees. However, the majority of the tested isolates were susceptible to colistin, indicating that this drug may be a viable alternative for treating infections with CRPA. ${ }^{7,15}$ However, a limitation of colistin treatment has been its inability to achieve adequate plasma concentrations, especially for infections caused by organisms with MIC values $>0.5 \mu \mathrm{g} \mathrm{ml}^{-1} .{ }^{16}$ Our study demonstrated that the colistin MIC values for most of the evaluated isolates were $\leqslant 0.5 \mu \mathrm{g} \mathrm{ml}^{-1}$ in the combination therapy. Therefore, combination therapies may provide alternative treatment strategies and improve treatment outcomes.

Fosfomycin has demonstrated low toxicity against a number of infections and has a role in the treatment of infections caused by MDR-PA. Some studies have also reported that fosfomycin improves treatment outcomes, prevents antibiotic resistance, and reduces the nephrotoxicity induced by aminoglycoside or vancomycin. ${ }^{17-20}$ In this study, colistin and fosfomycin displayed synergistic and partial synergistic effects in $49.43 \%$ of the isolates, and no antagonism was observed. The results were partially consistent with a previous study by Samonis et al., ${ }^{21}$ in which 15 MDR-PA isolates were tested. Based on the CIR curves, we observed a notable difference between single agent and combination treatment against CRPA isolates. In combination therapy, the fosfomycin and colistin MIC values for most of the isolates were significantly lower than the plasma concentrations that can be achieved for both drugs. ${ }^{22,23}$ Thus, fosfomycin and colistin could be used in combination when treating CRPA infections.

The time-kill assay results were consistent with those of the checkerboard method. Colistin alone at $1 / 2 \times$ MIC could not inhibit bacterial growth, and at $1 \times$ MIC produced minimal initial killing of PA-1, PA-2, PA-3, and PA-4 within $4 \mathrm{~h}$. This result suggests that the bactericidal activity of colistin is concentration dependent. However, the bacteriostatic activity of colistin was not sustained, and regrowth occurred rapidly after $4 \mathrm{~h}$ of treatment. The heteroresistance of the isolates might contribute to the observed regrowth following colistin monotherapy. ${ }^{24}$ When colistin was combined with fosfomycin, the treatment's bactericidal activity was substantially enhanced, and the regrowth that occurred with colistin monotherapy was prevented. Rao et al. ${ }^{25}$ have demonstrated that increasing the $C_{\max }$ of colistin monotherapy could not completely eliminate the bacteria population, but the addition of a synergistic second antimicrobial may sustain the initial killing achieved by a colistin front-loaded regimen. Enhanced bacterial killing was particularly evident for combinations at $1 / 2 \times \mathrm{MIC}$ against PA-1, PA-2, PA-3, and PA-4, and no viable bacteria was detected at $24 \mathrm{~h}$ (Figure 3 ). The time-kill assay demonstrated that the co-administration of fosfomycin possesses the potential to increase the therapeutic index of colistin.

The limitation of this study was the lack of molecular characterization and fingerprinting of the CRPA isolates. Epidemiological typing could provide additional insight into the resistance mechanisms of CRPA.
In conclusion, the combination of fosfomycin and colistin displayed significant synergistic activity against most of the tested CRPA isolates. The presence of fosfomycin enhanced and prolonged the bactericidal effects of colistin. Fosfomycin may widen colistin's therapeutic window, especially when treating an infection caused by organisms for which an MIC of $>0.5 \mu \mathrm{g} \mathrm{ml}^{-1}$ is required. Further research is needed to clarify the potential utility of the combination treatment of colistin and fosfomycin in vivo and in the clinic.

\section{CONFLICT OF INTEREST}

The authors declare no conflicts of interest.

\section{ACKNOWLEDGEMENTS}

This study was supported by the Major National Science and Technology Special Projects for New Drugs (No.2012ZX09303004) and the Beijing Municipal Natural Science Foundation (No.7122167).

1 Kollef, M. H. Appropriate empiric antimicrobial therapy of nosocomial pneumonia: the role of the carbapenems. Respir Care. 49, 1530-1541 (2004).

2 Lautenbach, E. et al. Imipenem resistance in Pseudomonas aeruginosa: emergence, epidemiology, and impact on clinical and economic outcomes. Infect. Control Hosp. Epidemiol. 31, 47-53 (2010).

3 Souli, M., Galani, I. \& Giamarellou, H. Emergence of extensively drug-resistant and pandrug-resistant Gram-negative bacilli in Europe. Euro Surveill. 13, 19045 (2008)

4 Giamarellou, H. \& Poulakou, G. Multidrug-resistant Gram-negative infections: what are the treatment options? Drugs 69, 1879-1901 (2009).

5 Falagas, M. E. \& Rafailidis, P. I. Re-emergence of colistin in today's world of multidrugresistant organisms: personal perspectives. Expert Opin. Investig. Drugs 17 973-981 (2008).

6 Michalopoulos, A. S. \& Karatza, D. C. Multidrug-resistant Gram-negative infections: the use of colistin. Expert Rev. Anti Infect. Ther. 8, 1009-1017 (2010).

7 Yahav, D., Farbman, L., Leibovici, L. \& Paul, M. Colistin: new lessons on an old antibiotic. Clin. Microbiol. Infect. 18, 18-29 (2012).

8 Petrosillo, N., Ioannidou, E. \& Falagas, M. E. Colistin monotherapy vs. combination therapy: evidence from microbiological, animal and clinical studies. Clin. Microbiol. Infect. 14, 816-827 (2008).

9 Karageorgopoulos, D. E., Wang, R., Yu, X. \& Falagas, M. E. Fosfomycin: Evaluation of the published evidence on the emergence of antimicrobial resistance in gram-negative pathogens. J. Antimicrob. Chemother. 67, 255-268 (2012).

10 Clinical and Laboratory Standards Insitute. Performance Standards for Antimicrobial Susceptebility Testing: Twenty-third Informational Supplement. Report No. M100-S23 (CLSI, Wayne, PA, USA, 2013).

11 Magiorakos, A. P. et al. Multidrug-resistant, extensively drug-resistant and pandrugresistant bacteria: an international expert proposal for interim standard definitions for acquired resistance. Clin. Microbiol. Infect. 18, 268-281 (2012).

12 Moody, J. in Clinical Microbiology Procedures Handbook (ed. Isenberg, H. D.) 1-28 (ASW, Washington, DC, USA, 2004).

13 White, R. L., Burgess, D. S., Manduru, M. \& Bosso, J. A. Comparison of three different in vitro methods of detecting synergy: time-kill, checkerboard, and E test. Antimicrob. Agents Chemother. 40, 1914-1918 (1996).

14 Galani, I., Orlandou, K., Moraitou, H., Petrikkos, G. \& Souli, M. Colistin/ daptomycin: an unconventional antimicrobial combination synergistic in vitro against multidrug resistant Acinetobacter baumannii. Int. J. Antimicrob. Agents 43, 370-374 (2014).

15 Crusio, R. et al. Epidemiology and outcome of infections with carbapenem -resistant Gram-negative bacteria treated with polymyxin B-based combination therapy. Scand. J. Infect. Dis. 46, 1-8 (2014).

16 Garonzik, S. M. et al. Population pharmacokinetics of colistin methanesulfonate and formed colistin in critically ill patients from a multicenter study provide dosing suggestions for various categories of patients. Antimicrob. Agents Chemother. 55, 3284-3294 (2011).

17 McCaughey, G., Diamond, P., Elborn, J.S., McKevitt, M. \& Tunney, M. M. Resistance development of cystic fibrosis respiratory pathogens when exposed to fosfomycin and tobramycin alone and in combination under aerobic and anaerobic conditions. PLOS ONE 8, e69763 (2013).

18 Nakamura, T., Kokuryo, T., Hashimoto, Y. \& Inui, K. I. Effects of fosfomycin and imipenem-cilastatin on the nephrotoxicity of vancomycin and cisplatin in rats. J. Pharm. Pharmacol. 51, 227-232 (1999).

19 Yanagida, C., Ito, K., Komiya, I. \& Horie, T. Protective effect of fosfomycin on gentamicin- induced lipid peroxidation of rat renal tissue. Chem. Biol. Interact. 148, 139-147 (2004).

20 Sahuquillo Arce, J.M. et al. In vitro activity of linezolid in combination with doxycycline, fosfomycin, levofloxacin, rifampicin and vancomycin against methicillin-susceptible Staphylococcus aureus. Rev. Esp. Quimioter. 19, 252-257 (2006). 
21 Samonis, G., Maraki, S., Karageorgopoulos, D. E., Vouloumanou, E. K. \& Falagas, M.E. Synergy of fosfomycin with carbapenems, colistin, netilmicin, and tigecycline against multidrug-resistant Klebsiella pneumoniae, Escherichia coli, and Pseudomonas aeruginosa clinical isolates. Eur. J. Clin. Microbiol. Infect. Dis. 31, 695-701 (2012).

22 Markou, N. et al. Colistin serum concentrations after intravenous administration in critically ill patients with serious multidrug-resistant, Gram-negative bacilli infections: a prospective, open-label, uncontrolled study. Clin. Ther. 30, 143-151 (2008).

23 Roussos, N., Karageorgopoulos, D. E., Samonis, G. \& Falagas, M. E. Clinical significance of the pharmacokinetic and pharmacodynamic characteristics of fosfomycin for the treatment of patients with systemic infections. Int. J. Antimicrob. Agents 34, 506-515 (2009).

24 Bergen, P. J. et al. Clinically relevant plasma concentrations of colistin in combination with imipenem enhance pharmacodynamic activity against multidrug-resistant Pseudomonas aeruginosa at multiple inocula. Antimicrob. Agents Chemother. 55, 5134-5142 (2011).

25 Rao, G.G. et al. New dosing strategies for an old antibiotic: pharmacodynamics of frontloaded regimens of colistin at simulated pharmacokinetics in patients with kidney or liver disease. Antimicrob. Agents Chemother. 58, 1381-1388 (2014). 